\title{
MEAN PERCENTAGE IMPROVEMENT IN PSORIASIS AREA AND SEVERITY INDEX (PASI) RESPONSE AND ABSOLUTE PASI THROUGH 5 YEARS OF CONTINUOUS TREATMENT WITH GUSELKUMAB IN VOYAGE 1
}

Joseph F. Merola ${ }^{1}$, Luis Puig², Megan Miller ${ }^{3}$, Yin You ${ }^{3}$, Yaung-Kaung Shen ${ }^{3}$, Ya-Wen Yang ${ }^{4}$, Andrew Blauvelt ${ }^{5}$

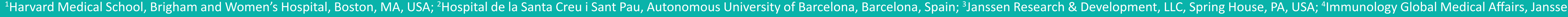
Pharmaceutical Companies of Johnson \& Johnson, Horsham, PA, USA; '5Oregon Medical Research Center, Portland, OR, USA

\begin{tabular}{|c|}
\hline BACKGROUND/OBJECTIVE \\
\hline 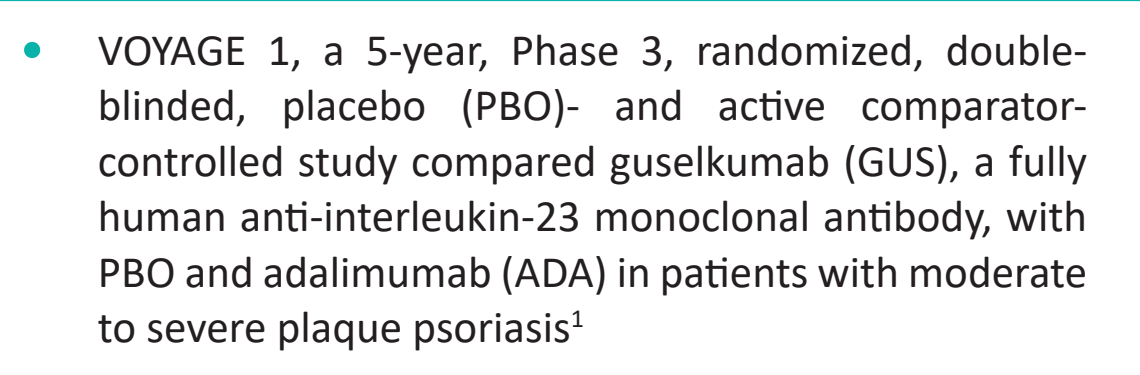 \\
\hline 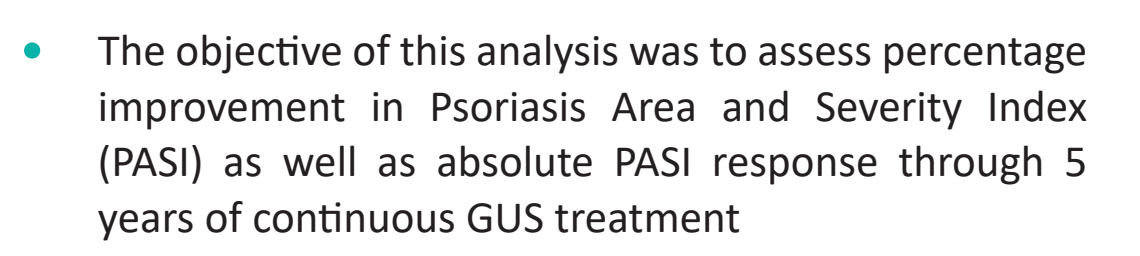 \\
\hline
\end{tabular}

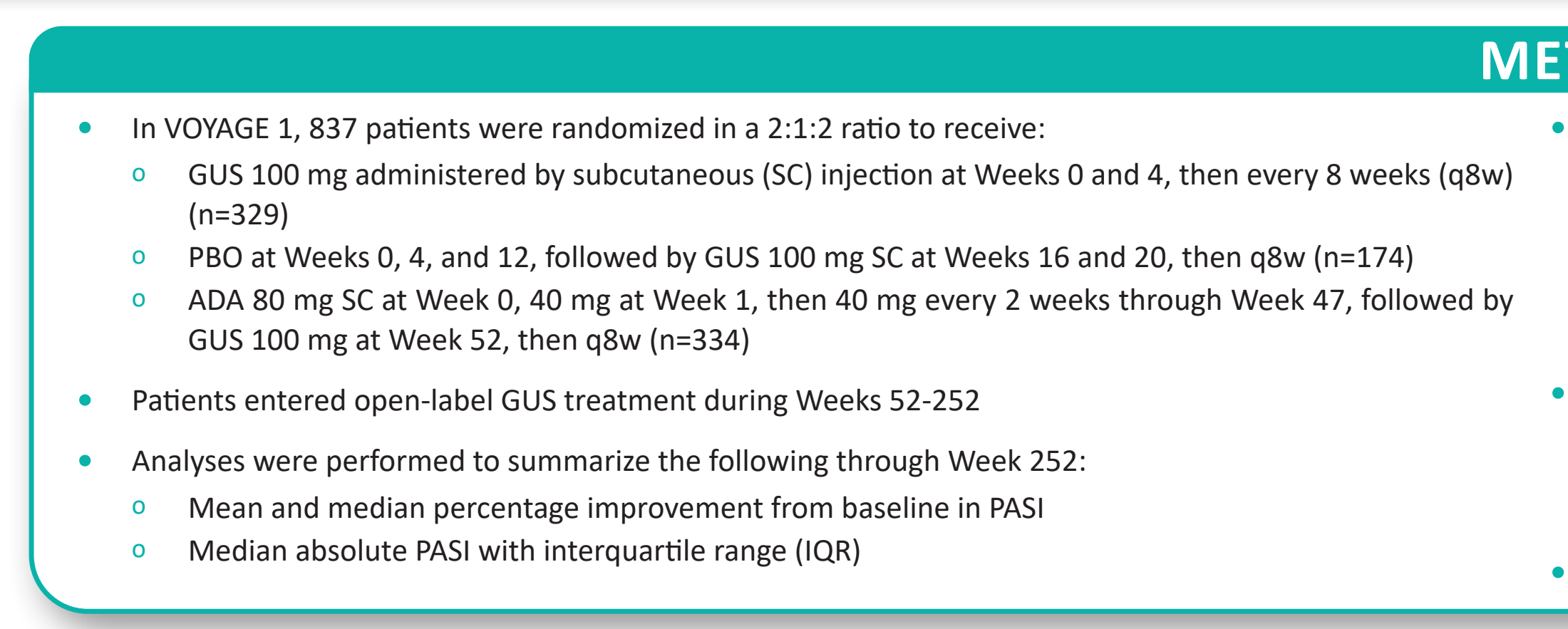

METHODS

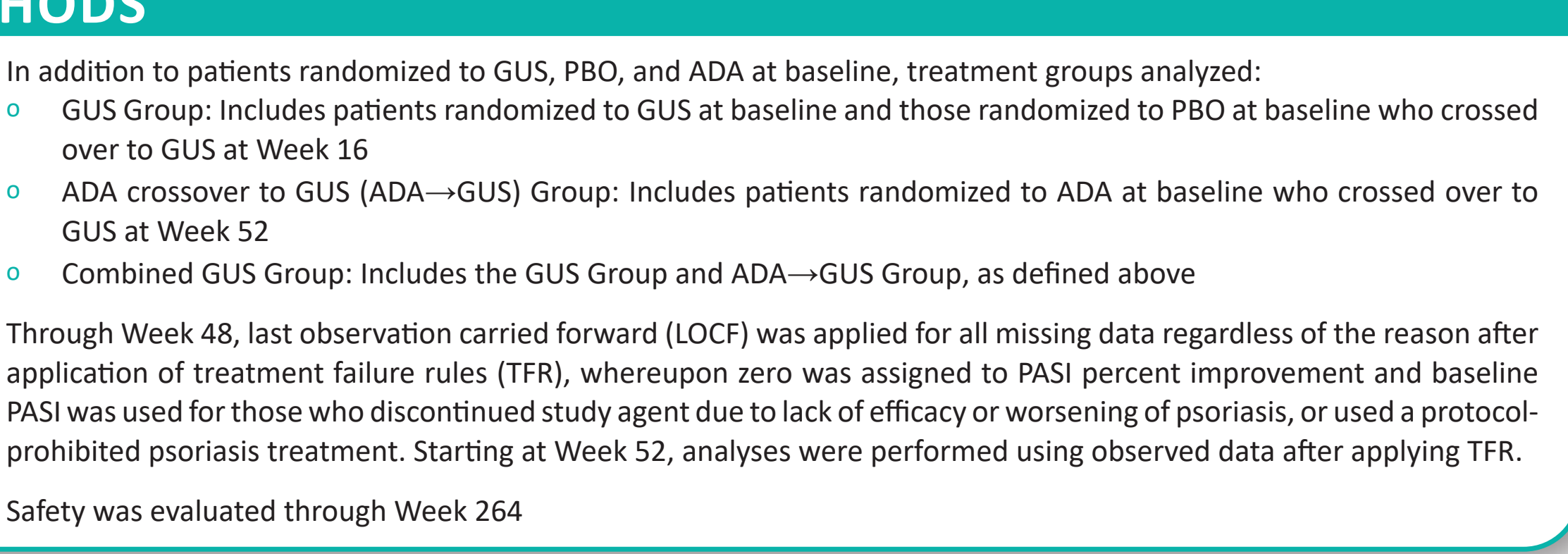

CONCLUSIONS

Continuous treatment with GUS provided robust and durable skin responses based on percentage improvement in PASI as well as absolute PASI through 5 years

No new safety concerns were identified through Week 264

\begin{tabular}{|c|c|c|c|c|}
\hline \multicolumn{5}{|c|}{ 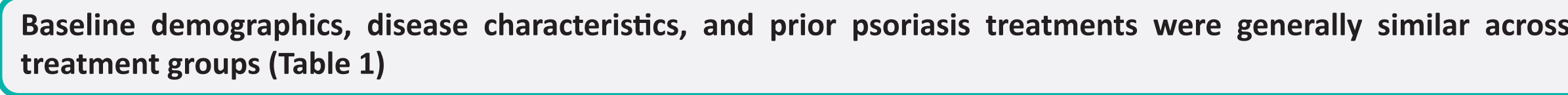 } \\
\hline \multicolumn{5}{|c|}{ Table 1. Baseline Demographics and Disease Characteristics } \\
\hline & PBO & GUS & ADA & Total \\
\hline Randomized patients, n & 174 & 329 & 334 & 837 \\
\hline Age [years] & $44.9 \pm 12.9$ & $43.9 \pm 12.7 \mathrm{P}-2$ & $42.9 \pm 12.6 \mathrm{P}-2 \mathrm{C}$ & $43.7 \pm 12.7$ \\
\hline Male, n (\%) & $119(68.4)$ & $240(72.9)$ & $249(74.6)$ & $608(72.6)$ \\
\hline Body mass index $\left(\mathrm{kg} / \mathrm{m}^{2}\right)$ & $28.9 \pm 6.9$ & $29.7 \pm 6.2$ & $29.8 \pm 6.5$ & $29.6 \pm 6.5$ \\
\hline Body surface area (\%) & $25.8 \pm 15.9$ & $28.3 \pm 17.1$ & $28.6 \pm 16.7$ & $27.9 \pm 16.7$ \\
\hline PASI (0-72) & $20.4 \pm 8.7$ & $22.1 \pm 9.5$ & $22.4 \pm 9.0$ & $21.9 \pm 9.2$ \\
\hline IGA score (moderate), \% & 75.3 & 76.6 & 72.2 & 74.6 \\
\hline IGA score (severe), \% & 24.7 & 23.4 & 26.9 & 25.1 \\
\hline Duration of psoriasis [years] & $17.6 \pm 12.4$ & $17.9 \pm 12.3$ & $17.0 \pm 11.3$ & $17.5 \pm 11.9$ \\
\hline Patients with psoriatic arthritis, n $(\%)$ & $30(17.2)$ & $64(19.5)$ & $62(18.6)$ & 156 (18.6) \\
\hline \multicolumn{5}{|l|}{ Prior psoriasis treatments, $n$ (\%) } \\
\hline Topical agents & $154(88.5)$ & $299(90.9)$ & $30992.8)$ & $762(91.1)$ \\
\hline Phototherapy (PUVA or UVB) & $86(49.4)$ & $188(57.3)$ & $180(53.9)$ & $454(54.3)$ \\
\hline Non-biologic systemics & 92(52.9) & $210(63.8)$ & 215 (64.4) & $517(61.8)$ \\
\hline Biologics & $34(19.5)$ & $71(21.6)$ & $70(22.0)$ & $175(20.9)$ \\
\hline
\end{tabular}

Mean percentage improvement from baseline in PASI was $93 \%$ or reater at each time point in the GUS group fir
Week 52 through Week 252 . A similar response was observed in the ADA $\rightarrow$ GUS group over time. (Figure 1 )

Median percentage improvement from baseline in PASl over time is shown in Figure 2

Figure 1 . Mean Percent

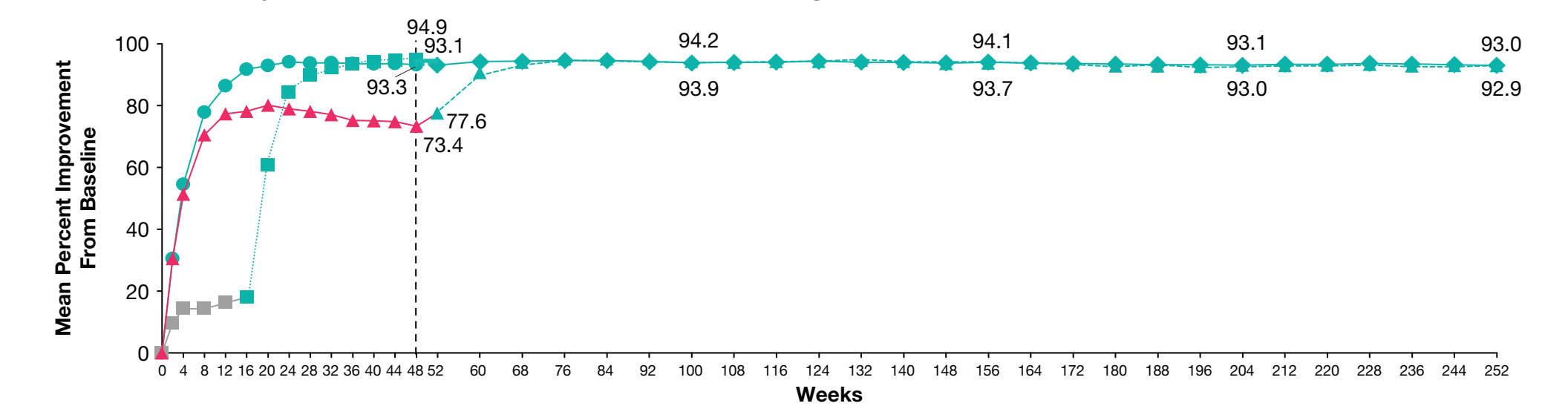

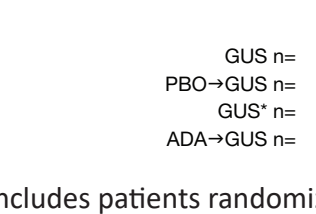

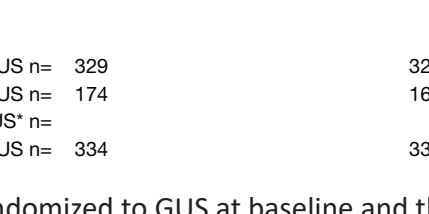

Figure 2. Median Percent Improvement From Baseline in PASI (IRR) Through Week 252

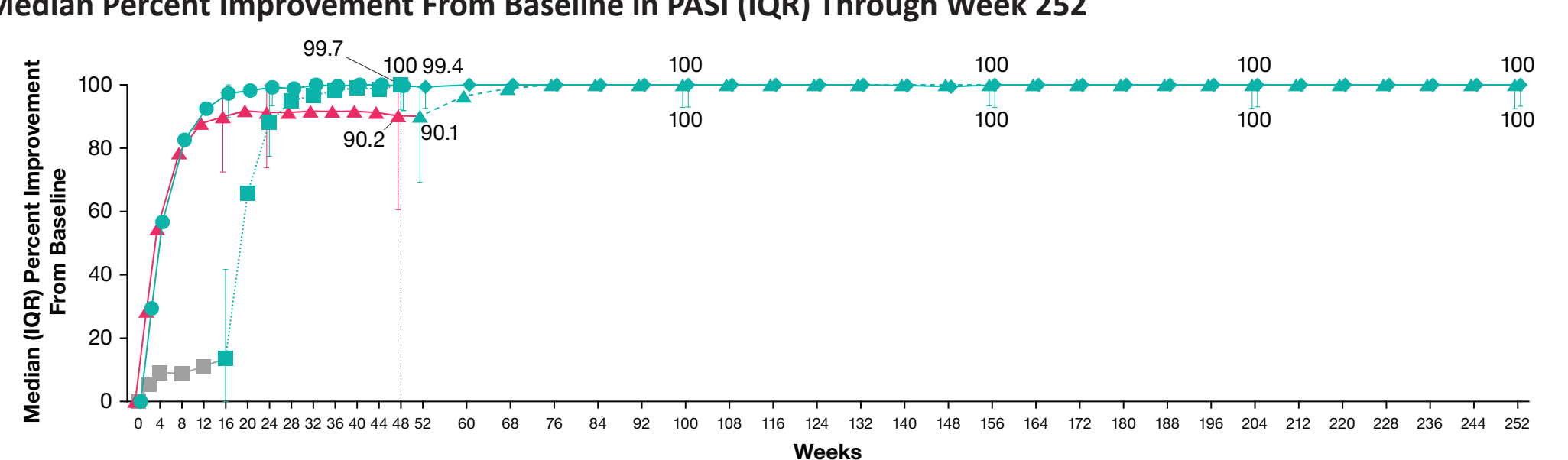

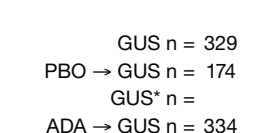

Median absolute PASI (IOR) was $0.10(0.00 ; 1.65)$ in the GUS group $(n=468)$ and $2.00(0.20 ; 6.00)$ in the ADA $\rightarrow$ GUS group $(n=279)$ at Week 52; ans
group $(n=246)$ at Week 252 Figure 3. Median Absolute PASI (IRR) Through Week 252
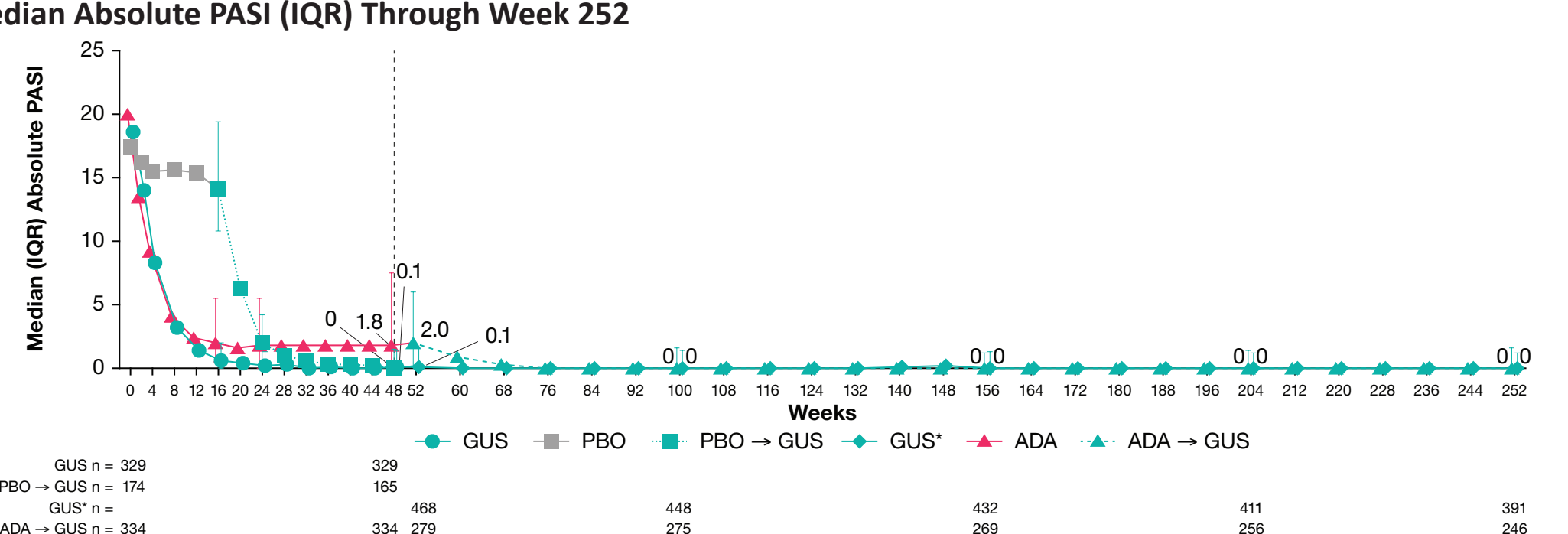

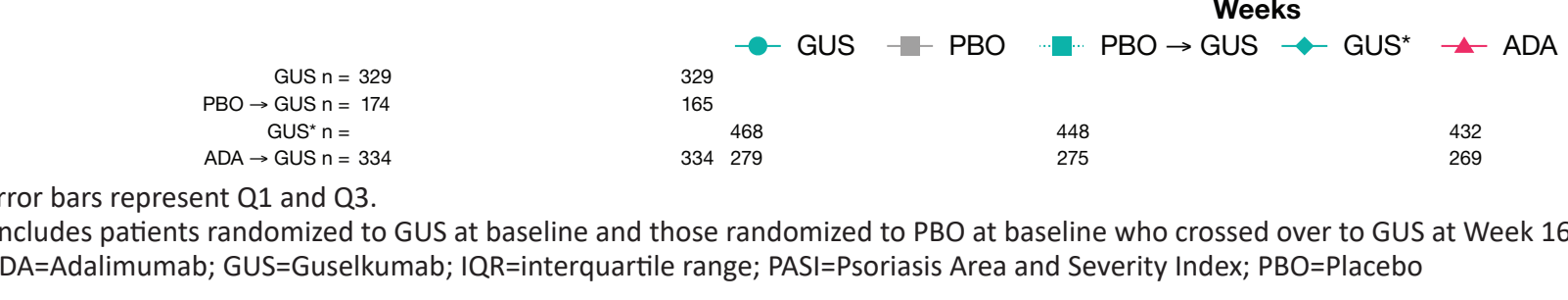

No new safety signals were identified through Week 264 (Table 2) Table 2. Adverse Events Through Week 264
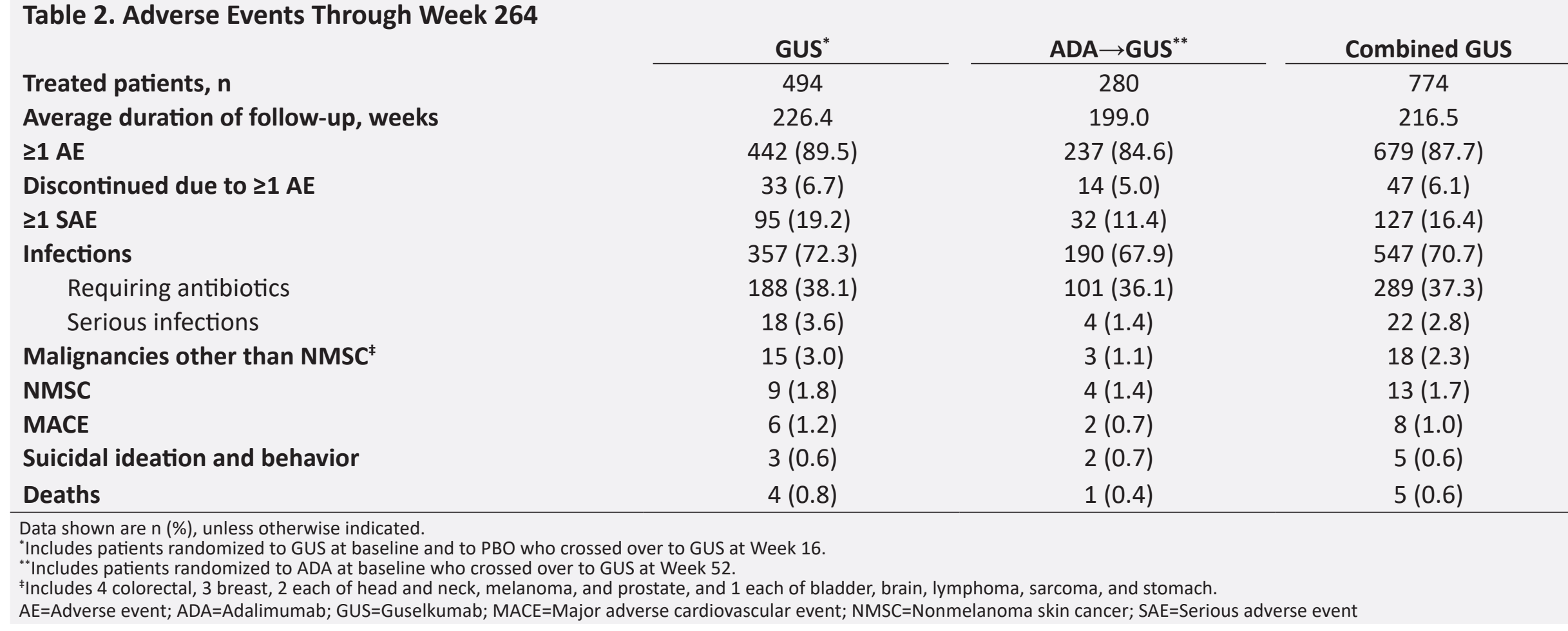

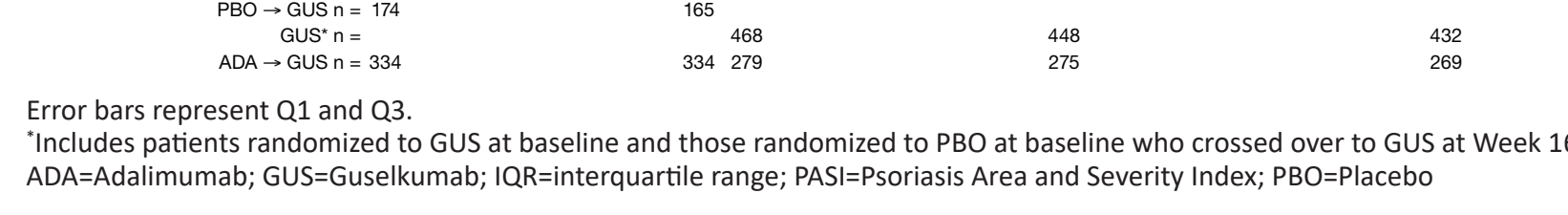

